\title{
ON THE POLYNOMIAL INTEGRABILITY OF A SYSTEM MOTIVATED BY THE RIEMANN ELLIPSOID PROBLEM
}

\author{
JAUME LLIBRE ${ }^{1}$ AND CLÀUDIA VALLS ${ }^{2}$
}

\begin{abstract}
We consider differential systems obtained by coupling two Euler-Poinsot systems. The motivation to consider such systems can be traced back to the Riemann ellipsoid problem. We provide new cases for which these systems are completely integrable. We also prove that these systems either are completely integrable or have at most four functionally independent polynomial first integrals.
\end{abstract}

\section{IntRoduCtion AND STATEMENT OF THE MAIN RESUlts}

Consider the following system of differential equations

$$
\begin{aligned}
& \frac{d x}{d t}=\nabla_{x} G(x, y) \wedge x, \\
& \frac{d y}{d t}=\nabla_{y} G(x, y) \wedge y,
\end{aligned}
$$

where $(x, y) \in \mathbb{R}^{3} \times \mathbb{R}^{3}$ and $G$ is the quadratic form

$$
G=\frac{1}{2} \sum_{i=1}^{3}\left(a_{i}\left(x_{i}^{2}+y_{i}^{2}\right)+2 b_{i} x_{i} y_{i}\right)
$$

The $a_{i}, b_{i}, i=1,2,3$ are real constants. To avoid the trivial cases, at least one of the coupling constants $b_{i}$ 's is assumed to be different from zero. Of course, $x=$ 0 (or $y=0$ ) is an invariant subspace and here system (1) reduces to the EulerPoinsot equations. The motivation to consider such systems can be traced back to the Riemann ellipsoid problem, see for more details $[3,5,6]$.

Expanding the notation, system (1) writes as

$$
\begin{aligned}
& \dot{x}_{1}=\left(a_{2}-a_{3}\right) x_{2} x_{3}+b_{2} x_{3} y_{2}-b_{3} x_{2} y_{3}=P_{1}\left(x_{1}, x_{2}, x_{3}, y_{1}, y_{2}, y_{3}\right), \\
& \dot{x}_{2}=\left(a_{3}-a_{1}\right) x_{1} x_{3}+b_{3} x_{1} y_{3}-b_{1} x_{3} y_{1}=P_{2}\left(x_{1}, x_{2}, x_{3}, y_{1}, y_{2}, y_{3}\right), \\
& \dot{x}_{3}=\left(a_{1}-a_{2}\right) x_{1} x_{2}+b_{1} x_{2} y_{1}-b_{2} x_{1} y_{2}=P_{3}\left(x_{1}, x_{2}, x_{3}, y_{1}, y_{2}, y_{3}\right), \\
& \dot{y}_{1}=b_{2} x_{2} y_{3}-b_{3} x_{3} y_{2}+\left(a_{2}-a_{3}\right) y_{2} y_{3}=P_{4}\left(x_{1}, x_{2}, x_{3}, y_{1}, y_{2}, y_{3}\right), \\
& \dot{y}_{2}=b_{3} x_{3} y_{1}-b_{1} x_{1} y_{3}+\left(a_{3}-a_{1}\right) y_{1} y_{3}=P_{5}\left(x_{1}, x_{2}, x_{3}, y_{1}, y_{2}, y_{3}\right), \\
& \dot{y}_{3}=b_{1} x_{1} y_{2}-b_{2} x_{2} y_{1}+\left(a_{1}-a_{2}\right) y_{1} y_{2}=P_{6}\left(x_{1}, x_{2}, x_{3}, y_{1}, y_{2}, y_{3}\right),
\end{aligned}
$$

2010 Mathematics Subject Classification. Primary 34C05, 34A34, 34C14.

Key words and phrases. Polynomial first integrals, homogeneous differential systems, Riemmann ellipsoid problem. 
where $\left(x_{1}, x_{2}, x_{3}, y_{1}, y_{2}, y_{3}\right) \in \mathbb{R}^{6}$ and $a_{i}, b_{i} \in \mathbb{R}$ for $i=1,2,3$ such that at least one $b_{i}$ is assumed to be different from zero.

It is immediate to verify that system (2) has the following polynomial three first integrals

$$
H_{1}=\sum_{i=1}^{3} x_{i}^{2}, \quad H_{2}=\sum_{i=1}^{3} y_{i}^{2}, \quad H_{3}=\sum_{i=1}^{3}\left[a_{i}\left(x_{i}^{2}+y_{i}^{2}\right)+2 b_{i} x_{i} y_{i}\right] .
$$

which are functionally independent. We recall that given $U$ an open set of $\mathbb{R}^{6}$ such that $\mathbb{R}^{6} \backslash U$ has zero Lebesgue measure, we say that a real function $H=$ $H\left(x_{1}, x_{2}, x_{3}, y_{1}, y_{2}, y_{3}\right): U \subset \mathbb{R}^{6} \rightarrow \mathbb{R}$ is a first integral if $H$ is constant for all values of a solution $\left(x_{1}(t), x_{2}(t), x_{3}(t), y_{1}(t), y_{2}(t), y_{3}(t)\right)$ of system $(2)$ contained in $U$, i.e. $H$ is a first integral in $U$ if and only if

$$
\sum_{i=1}^{3}\left(\frac{\partial H}{\partial x_{i}} P_{i}\left(x_{1}, x_{2}, x_{3}, y_{1}, y_{2}, y_{3}\right)+\frac{\partial H}{\partial y_{i}} P_{i+3}\left(x_{1}, x_{2}, x_{3}, y_{1}, y_{2}, y_{3}\right)\right)=0
$$

on the points of $U$. Moreover, the first integrals $H_{1}, \ldots, H_{r}$ are functionally independent if the $r \times 6$ matrix

$$
\left(\begin{array}{ccc}
\partial H_{1} / \partial x_{1} & \cdots & \partial H_{1} / \partial y_{3} \\
\vdots & \cdots & \vdots \\
\partial H_{r} / \partial x_{1} & \cdots & \partial H_{r} / \partial y_{3}
\end{array}\right)\left(x_{1}, x_{2}, x_{3}, y_{1}, y_{2}, y_{3}\right)
$$

has rank $r$ at all points $\left(x_{1}, x_{2}, x_{3}, y_{1}, y_{2}, y_{3}\right) \in \mathbb{R}^{6}$ where they are defined except perhaps in a zero Lebesgue measure set.

We are interested in finding additional polynomial first integrals which are functionally independent with $\mathrm{H}_{1}, \mathrm{H}_{2}$ and $H_{3}$.

We know that since system (2) has zero divergence it follows from Theorem 2.7 of [2] that if it has 4 functionally independent analytic first integrals then the system is completely integrable, i.e. it has 5 first integrals functionally independent first integrals.

We note that system (2) is invariant under the diffeomorphism

$$
\tau\left(x_{1}, x_{2}, x_{3}, y_{1}, y_{2}, y_{3}, a_{1}, a_{2}, a_{3}, b_{1}, b_{2}, b_{3}\right) \rightarrow\left(x_{2}, x_{3}, x_{1}, y_{2}, y_{3}, y_{1}, a_{2}, a_{3}, a_{1}, b_{2}, b_{3}, b_{1}\right)
$$

First we obtain some polynomial first integrals.

Theorem 1. The differential systems (2) have a fourth polynomial first integral $H_{4}$ functionally independent with $\mathrm{H}_{1}, \mathrm{H}_{2}$ and $\mathrm{H}_{3}$ if

(a) $b_{1}= \pm b_{2}$ and $a_{1}=a_{2}$, then $H_{4}= \pm x_{3}+y_{3}$;

(b) $b_{1}= \pm b_{3}$ and $a_{1}=a_{3}$, then $H_{4}= \pm x_{2}+y_{2}$;

(c) $b_{2}= \pm b_{3}$ and $a_{2}=a_{3}$, then $H_{4}= \pm x_{1}+y_{1}$;

(d) $b_{1}=a_{2}-a_{1}+b_{2}, b_{3}=a_{3}-a_{2}-b_{2}, a_{1} \neq a_{2}$ and $a_{3} \neq a_{2}$, then $H_{4}=$ $\left(x_{1}+y_{1}\right)^{2}+\left(x_{2}+y_{2}\right)^{2}+\left(x_{3}-y_{3}\right)^{2}$

(e) $b_{1}=a_{1}-a_{2}+b_{2}, b_{3}=a_{3}-a_{2}+b_{2}, a_{1} \neq a_{2}$ and $a_{3} \neq a_{2}$, then $H_{4}=$ $\left(x_{1}-y_{1}\right)^{2}+\left(x_{2}-y_{2}\right)^{2}+\left(x_{3}-y_{3}\right)^{2}$; 
(f) $b_{1}=a_{2}-a_{1}-b_{2}, b_{3}=a_{2}-a_{3}-b_{2}, a_{1} \neq a_{2}$ and $a_{3} \neq a_{2}$, then $H_{4}=$ $\left(x_{1}+y_{1}\right)^{2}+\left(x_{2}-y_{2}\right)^{2}+\left(x_{3}+y_{3}\right)^{2}$

(g) $b_{1}=a_{1}-a_{2}-b_{2}, b_{3}=a_{2}-a_{3}+b_{2}, a_{1} \neq a_{2}$ and $a_{2} \neq a_{3}$, then $H_{4}=$ $\left(x_{1}-y_{1}\right)^{2}+\left(x_{2}+y_{2}\right)^{2}+\left(x_{3}+y_{3}\right)^{2}$

(h) $b_{1}=a_{1}-a_{2}+b_{2}, b_{3}=a_{2}-a_{3}-b_{2}, a_{1} \neq a_{2}$ and $a_{3} \neq a_{2}$, then $H_{4}=$ $\frac{a_{2}-a_{3}-b_{3}}{a_{2}-a_{3}}\left(x_{1}-y_{1}\right)^{2}+\frac{a_{1}-a_{3}-b_{3}}{a_{1}-a_{3}}\left(x_{2}-y_{2}\right)^{2}+\left(x_{3}-y_{3}\right)^{2}$

(i) $b_{1}=a_{2}-a_{1}-b_{2}, b_{3}=a_{3}-a_{2}+b_{2}, a_{1} \neq a_{2}$ and $a_{3} \neq a_{2}$, then $H_{4}=$ $-\frac{a_{1}-a_{2}+b_{1}}{a_{2}-a_{3}}\left(x_{1}+y_{1}\right)^{2}-\frac{b_{1}}{a_{1}-a_{3}}\left(x_{2}-y_{2}\right)^{2}+\left(x_{3}+y_{3}\right)^{2}$

(j) $b_{1}=a_{2}-a_{1}+b_{2}, b_{3}=a_{2}-a_{3}+b_{2}, a_{1} \neq a_{2}$ and $a_{2} \neq a_{3}$, then $H_{4}=$ $-\frac{b_{2}}{a_{2}-a_{3}}\left(x_{1}+y_{1}\right)^{2}+\frac{a_{1}-a_{2}-b_{2}}{a_{1}-a_{3}}\left(x_{2}+y_{2}\right)^{2}+\left(x_{3}-y_{3}\right)^{2}$

(k) $b_{1}=a_{1}-a_{2}-b_{2}, b_{3}=a_{3}-a_{2}-b_{2}, a_{1} \neq a_{2}$ and $a_{2} \neq a_{3}$, then $H_{4}=$ $-\frac{b_{2}}{a_{2}-a_{3}}\left(x_{1}-y_{1}\right)^{2}+\frac{a_{1}-a_{2}-b_{2}}{a_{1}-a_{3}}\left(x_{2}+y_{2}\right)^{2}+\left(x_{3}+y_{3}\right)^{2}$.

The cases of integrability of Theorem 1 were already known by Negrini (see Theorems 2 and 3 of [6]), but he did not know that the fourth functionally independent first integral of systems (2) of the statements (a), (b) and (c) of Theorem 1 can be polynomial.

Theorem 1 can be checked easily by direct computations.

Corollary 2. The differential systems (2) satisfying the conditions of Theorem 1 are completely integrable.

Corollary 2 is proved in section 2, but it was also known by Negrini in [6].

Theorem 3. The differential systems (2) either satisfy the conditions of Theorem 1, or have at most four functionally independent polynomial first integrals.

Theorem 3 is proved in section 3 .

In [6] the author also gaves conditions for the existence or nonexistence of meromorphic first integrals for system (2).

\section{Proof of Corollary 2}

The following result is due to Jacobi. For a proof in a more general setting see Theorem 2.7 of [2].

Theorem 4. Consider an analytic differential system in $\mathbb{R}^{n}$ of the form

$$
\frac{d x}{d t}=\dot{x}=P(x), \quad x=\left(x_{1}, \ldots, x_{n}\right) \in \mathbb{R}^{n}
$$

with $P(x)=\left(P_{1}(x), \ldots, P_{n}(x)\right)$. Assume that

$$
\sum_{i=1}^{n} \frac{\partial P_{i}}{\partial x_{i}}=0 \quad \text { (i.e. it has zero divergence) }
$$


and that it admits $n-2$ first integrals, $I_{i}(x)=c_{i}$ with $i=1, \ldots, n-2$ functionally independent. These integrals define, up to a relabeling of the variables, an invertible transformation mapping from $\left(x_{1}, \ldots, x_{n}\right)$ to $\left(c_{1}, \ldots, c_{n-2}, x_{n-1}, x_{n}\right)$ given by

$$
y_{i}=I_{i}(x), \quad i=1, \ldots, n-2, \quad y_{n-1}=x_{n-1}, \quad y_{n}=x_{n} .
$$

Let $\Delta$ be the Jacobian of the transformation

$$
\Delta=\operatorname{det}\left(\begin{array}{cccc}
\partial_{x_{1}} I_{1} & \partial_{x_{2}} I_{1} & \cdots & \partial_{x_{n-2}} I_{1} \\
\partial_{x_{1}} I_{2} & \partial_{x_{2}} I_{2} & \cdots & \partial_{x_{n-2}} I_{2} \\
\vdots & \vdots & \ddots & \vdots \\
\partial_{x_{1}} I_{n-2} & \partial_{x_{2}} I_{n-2} & \cdots & \partial_{x_{n-2}} I_{n-2}
\end{array}\right)
$$

Then system (3) admits an extra first integral given by

$$
I_{n-1}=\int \frac{1}{\tilde{\Delta}}\left(\tilde{P}_{n} d x_{n-1}-\tilde{P}_{n-1} d x_{n}\right),
$$

where the tilde denotes the quantities expressed in the variables $\left(c_{1}, \ldots, c_{n-2}, x_{n-1}, x_{n}\right)$. Moreover this first integral is functionally independent with the previous $n-2$ first integrals, that is, the system is completely integrable.

Proof of Theorem 2. It is immediate to verify that the differential systems (2) in $\mathbb{R}^{6}$ have zero divergence because every $P_{i}$ does not depend on $x_{i}$ for $i=1,2,3$, and $P_{i}$ does not depend on $y_{i-3}$ for $i=4,5,6$. In the case of the conditions given in Theorem 1 the differential systems (2) have $4=6-2$ first integrals functionally independent. So in this case they satisfy the assumptions of Theorem 4. Therefore this case is completely integrable.

\section{Proof of Theorem 3}

We denote by $\mathbb{Z}_{+}$the set of non-negative integers. The following result, due to Zhang [7], will be used in a strong way in the proof of Theorem 3.

Theorem 5. For an analytic vector field $\mathcal{X}$ defined in a neighborhood of the origin in $\mathbb{R}^{n}$ associated to system (3) with $P(0)=0$, let $\lambda_{1}, \ldots, \lambda_{n}$ be the eigenvalues of $D P(0)$. Set

$$
G=\left\{\left(k_{1}, \ldots, k_{n}\right) \in\left(\mathbb{Z}_{+}\right)^{n}: \sum_{i=1}^{n} k_{i} \lambda_{i}=0, \sum_{i=1}^{n} k_{i}>0\right\} .
$$

Assume that system (3) has $r<n$ functionally independent analytic first integrals $\Phi_{1}(x), \ldots, \Phi_{r}(x)$ in a neighborhood of the origin. If the $\mathbb{Z}$-linear space generated by $G$ has dimension $r$, then any nontrivial analytic first integral of system (3) in a neighborhood of the origin is an analytic function of $\Phi_{1}(x), \ldots, \Phi_{r}(x)$.

Extensions of Theorem 5 can be found in $[1,4]$.

We call each element $\left(k_{1}, \ldots, k_{n}\right) \in G$ a resonant lattice of the eigenvalues $\lambda_{1}, \ldots, \lambda_{n}$.

Direct calculations show that the differential systems (2) have seven planes of singularities, but we only use for proving our result two of these planes of singularities. 
At the singularity $S_{1}=\left(0, x_{2}, 0,0, y_{2}, 0\right)$, the 6 -tuple of eigenvalues $\lambda=\left(\lambda_{1}, \ldots, \lambda_{6}\right)$ of the linear part of the differential systems (2) are

$$
\lambda=\left(0,0,-\sqrt{\frac{A_{1}-\sqrt{B_{1}}}{2}}, \sqrt{\frac{A_{1}-\sqrt{B_{1}}}{2}},-\sqrt{\frac{A_{1}+\sqrt{B_{1}}}{2}}, \sqrt{\frac{A_{1}+\sqrt{B_{1}}}{2}}\right)
$$

where

$$
\begin{aligned}
& A_{1}=\left(\left(a_{1}-a_{2}\right)\left(a_{2}-a_{3}\right)-b_{2}^{2}\right)\left(x_{2}^{2}+y_{2}^{2}\right)+2\left(\left(a_{1}-2 a_{2}+a_{3}\right) b_{2}-b_{1} b_{3}\right) x_{2} y_{2}, \\
& B_{1}=A_{1}^{2}-4 \Delta_{1},
\end{aligned}
$$

with

$$
\begin{aligned}
\Delta_{1}= & \left(\left(a_{2}-a_{1}\right) b_{2}\left(x_{2}^{2}+y_{2}^{2}\right)+\left(\left(a_{1}-a_{2}\right)^{2}+b_{2}^{2}-b_{1}^{2}\right) x_{2} y_{2}\right) \\
& \left(\left(a_{2}-a_{3}\right) b_{2}\left(x_{2}^{2}+y_{2}^{2}\right)+\left(\left(a_{2}-a_{3}\right)^{2}+b_{2}^{2}-b_{3}^{2}\right) x_{2} y_{2}\right) .
\end{aligned}
$$

From Theorem 5 we know that the number of functionally independent analytic first integrals of the differential systems (2) in a neighborhood of the singularities $S$ is at most the number of linearly independent elements of the set

$$
G_{1}=\left\{\left(k_{1}, \ldots, k_{6}\right) \in\left(\mathbb{Z}_{+}\right)^{6}: \sum_{i=1}^{6} k_{i} \lambda_{i}=0, \sum_{i=1}^{6} k_{i}>0\right\} .
$$

According to the eigenvalues (4) the resonant lattices satisfy

$$
\sqrt{A_{1}-\sqrt{B_{1}}}\left(k_{4}-k_{3}\right)+\sqrt{A_{1}+\sqrt{B_{1}}}\left(k_{6}-k_{5}\right)=0 .
$$

This last equation has the following linearly independent non-negative solutions $\left(k_{1}, \ldots, k_{6}\right)$ :

$$
(1,0,0,0,0,0),(0,1,0,0,0,0),(0,0,1,1,0,0) \text { and }(0,0,0,0,1,1) .
$$

In order that equation (5) has an additional linearly independent non-negative integer solutions different from the above list, we must have

(i) either $\left(A_{1}-\sqrt{B_{1}}\right)\left(A_{1}+\sqrt{B_{1}}\right)=0$;

(ii) or $\left(A_{1}-\sqrt{B_{1}}\right)\left(A_{1}+\sqrt{B_{1}}\right) \neq 0$ and $\sqrt{A_{1}-\sqrt{B_{1}}} / \sqrt{A_{1}+\sqrt{B_{1}}}$ is a rational number. Then $\Delta_{1} \neq 0$ and $A_{1} \neq 0$ (otherwise $\sqrt{-\sqrt{B_{1}}} / \sqrt{\sqrt{B_{1}}}$ cannot be a rational number). Set

$$
\frac{\sqrt{A_{1}-\sqrt{B_{1}}}}{\sqrt{A_{1}+\sqrt{B_{1}}}}=\frac{m}{n}, \quad m, n \in \mathbb{Z} \backslash\{0\} \text { coprime. }
$$

This last equality can be written in an equivalent way as

$$
\frac{\Delta_{1}}{A_{1}^{2}}=\frac{m^{2} n^{2}}{\left(m^{2}+n^{2}\right)^{2}}
$$

where we have used the fact that $B_{1}=A_{1}^{2}-4 \Delta_{1}$. 
In case (i) we obtain the following independent conditions:

$$
\begin{aligned}
& b_{1}= \pm b_{2}, a_{1}=a_{2} ; \\
& b_{2}= \pm b_{3}, a_{2}=a_{3} ; \\
& b_{1}= \pm\left(a_{1}-a_{2}\right), b_{2}=0 ; \\
& b_{3}= \pm\left(a_{2}-a_{3}\right), b_{2}=0 .
\end{aligned}
$$

In the first four cases we are inside the conditions of Theorem 1. Now we shall consider the last four cases. We denote them by

$$
s_{1,2}=\left\{b_{1}= \pm\left(a_{1}-a_{2}\right), b_{2}=0\right\}, \quad s_{3,4}=\left\{b_{3}= \pm\left(a_{2}-a_{3}\right), b_{2}=0\right\} .
$$

Lemma 6. The differential systems (2) under one of the conditions $s_{1}, s_{2}, s_{3}$ or $s_{4}$, either satisfy the conditions of Theorem 1, or the eigenvalues of the singularity $S_{2}=\left(x_{1}, 0,0, y_{1}, 0,0\right)$ do not have a fifth linearly independent resonant lattice.

Proof. At the singularity $S_{2}=\left(x_{1}, 0,0, y_{1}, 0,0\right)$, the 6 -tuple of eigenvalues of the linear part of the differential systems (2) are given by

$$
\lambda=\left(0,0,-\sqrt{\frac{A_{2}-\sqrt{B_{2}}}{2}}, \sqrt{\frac{A_{2}-\sqrt{B_{2}}}{2}},-\sqrt{\frac{A_{2}+\sqrt{B_{2}}}{2}}, \sqrt{\frac{A_{2}+\sqrt{B_{2}}}{2}}\right),
$$

where

$$
\begin{aligned}
& A_{2}=-\left(\left(a_{1}-a_{2}\right)\left(a_{1}-a_{3}\right)+b_{1}^{2}\right)\left(x_{1}^{2}+y_{1}^{2}\right)+2\left(\left(a_{2}-2 a_{1}+a_{3}\right) b_{1}-b_{2} b_{3}\right) x_{1} y_{1}, \\
& B_{2}=A_{2}^{2}-4 \Delta_{2},
\end{aligned}
$$

with

$$
\begin{aligned}
\Delta_{2}= & \left(\left(a_{1}-a_{2}\right) b_{1}\left(x_{1}^{2}+y_{1}^{2}\right)+\left(\left(a_{1}-a_{2}\right)^{2}+b_{1}^{2}-b_{2}^{2}\right) x_{1} y_{1}\right) \\
& \left(\left(a_{1}-a_{3}\right) b_{1}\left(x_{1}^{2}+y_{1}^{2}\right)\left(\left(a_{1}-a_{3}\right)^{2}+b_{1}^{2}-b_{3}^{2}\right) x_{1} y_{1}\right) .
\end{aligned}
$$

Now direct calculations show that under one of the conditions $s_{1}, s_{2}, s_{3}, s_{4}$, the equation $\Delta_{2}=0$ yields that either $b_{1}= \pm b_{3}, a_{1}=a_{3}$ and $b_{2}=0$; or $b_{2}=b_{1}=0$ and $a_{1}=a_{2}$; or $b_{1}=b_{2}=0$ and $b_{3}= \pm\left(a_{2}-a_{3}\right)= \pm\left(a_{1}-a_{3}\right)$. This last condition in fact splits into four different conditions. In all the cases we are under the conditions of Theorem 1. Then, under one of the conditions $s_{1}, s_{2}, s_{3}$ or $s_{4}$, either $\Delta_{2}=0$ and then we are under the conditions of Theorem 1 , or $\Delta_{2} \neq 0$. Now, working in a similar way as we did for the singularities $S_{1}$ for studying if there is a fifth linearly independent resonant lattice at $S_{1}$, we need to check if $\sqrt{A_{2}-\sqrt{B_{2}}} / \sqrt{A_{2}+\sqrt{B_{2}}} \neq 0$ is a rational number. For $S_{2}$ under one of the conditions $s_{1}, s_{2}, s_{3}$ or $s_{4}$ we write

$$
\frac{\sqrt{A_{2}-\sqrt{B_{2}}}}{\sqrt{A_{2}+\sqrt{B_{2}}}}=\frac{m_{2}}{n_{2}}, \quad m_{2}, n_{2} \in \mathbb{Z} \backslash\{0\} \text { coprime. }
$$

This last equation can be written as

$$
\frac{\Delta_{2}}{A_{2}^{2}}=\frac{m_{2}^{2} n_{2}^{2}}{\left(n_{2}+m_{2}\right)^{2}}
$$


Clearly we have that $A_{2} \neq 0$, otherwise $\sqrt{A_{2}-\sqrt{B_{2}}} / \sqrt{A_{2}+\sqrt{B_{2}}}$ is not a rational number. $\Delta_{2}$ should be a square of $\left(a_{1}-a_{2}\right) b_{1}\left(x_{1}^{2}+y_{1}^{2}\right)+\left(\left(a_{1}-a_{2}\right)^{2}+b_{1}^{2}-b_{2}^{2}\right) x_{1} y_{1}$ or of $\left(a_{1}-a_{3}\right) b_{1}\left(x_{1}^{2}+y_{1}^{2}\right)+\left(\left(a_{1}-a_{3}\right)^{2}+b_{1}^{2}-b_{3}^{2}\right) x_{1} y_{1}$. Without loss of generality we can write it as

$$
\begin{aligned}
& \left(a_{1}-a_{2}\right) b_{1}\left(x_{1}^{2}+y_{1}^{2}\right)+\left(\left(a_{1}-a_{2}\right)^{2}+b_{1}^{2}-b_{2}^{2}\right) x_{1} y_{1} \\
& =L_{2}^{2}\left(\left(a_{1}-a_{3}\right) b_{1}\left(x_{1}^{2}+y_{1}^{2}\right)+\left(\left(a_{1}-a_{3}\right)^{2}+b_{1}^{2}-b_{3}^{2}\right) x_{1} y_{1}\right),
\end{aligned}
$$

and it is easy to check that $A_{2} /\left(\left(a_{1}-a_{3}\right) b_{1}\left(x_{1}^{2}+y_{1}^{2}\right)+\left(\left(a_{1}-a_{3}\right)^{2}+b_{1}^{2}-b_{3}^{2}\right) x_{1} y_{1}\right)$ is a constant. Set

$$
A_{2}=K_{2}\left(\left(a_{1}-a_{3}\right) b_{1}\left(x_{1}^{2}+y_{1}^{2}\right)+\left(\left(a_{1}-a_{3}\right)^{2}+b_{1}^{2}-b_{3}^{2}\right) x_{1} y_{1}\right) .
$$

Then, from (9) and (10) equating to zero the coefficients of the monomials in the variables $x_{1}$ and $y_{1}$ we have

$$
\begin{aligned}
-b_{1}\left(-a_{1}+a_{2}+L_{2}^{2}\left(a_{1}-a_{3}\right)\right. & =0, \\
\left(a_{1}-a_{2}\right)^{2}+b_{1}^{2}-b_{2}^{2}-L_{2}^{2}\left(\left(a_{1}-a_{3}\right)^{2}+b_{1}^{2}-b_{3}^{2}\right) & =0, \\
\left(a_{1}-a_{3}\right)\left(a_{2}-a_{1}\right)-b_{1}^{2}+b_{1} K_{2}\left(a_{3}-a_{1}\right) & =0, \\
2 b_{1}\left(a_{2}+a_{3}-2 a_{1}\right)-2 b_{2} b_{3}-K_{2}\left(\left(a_{1}-a_{3}\right)^{2}+b_{1}^{2}-b_{3}^{2}\right) & =0,
\end{aligned}
$$

where $L_{2} / K_{2}=m_{2} n_{2} /\left(n_{2}^{2}+m_{2}^{2}\right) \neq 0$. For the conditions $s_{1}$ and $s_{2}$ we have that the solutions of (11) are

$$
\begin{aligned}
& b_{3}= \pm\left(a_{1}-a_{3}\right), \quad a_{1}=a_{2} ; \\
& b_{3}= \pm \frac{L_{2}^{2}-1}{L_{2}^{2}}\left(a_{1}-a_{2}\right), \quad K= \pm\left(1+L_{2}^{2}\right), \quad a_{3}=a_{1}+\frac{a_{2}-a_{1}}{L_{2}^{2}},
\end{aligned}
$$

where the last condition corresponds in fact to four conditions. In all cases we are under the assumptions of Theorem 1 (note that in the last four cases we have in fact that $\left.b_{3}= \pm\left(a_{2}-a_{3}\right)\right)$. Finally, for the conditions $s_{3}$ and $s_{4}$ we have that the solutions of (11) are

$$
\begin{aligned}
& a_{2}=a_{1}, \quad b_{1}=0 \\
& b_{1}= \pm\left(a_{1}-a_{2}\right)= \pm\left(a_{1}-a_{3}\right), \quad K_{2}^{2}=4, \quad L_{2}^{2}=1 \\
& b_{1}= \pm\left(a_{1}-a_{2}\right), \quad K_{2}=\mp\left(1+L_{2}^{2}\right), \quad a_{3}=a_{1} \mp \frac{b_{1}}{L_{2}^{2}}
\end{aligned}
$$

where every one of the two last conditions correspond in fact to two conditions. In all cases we are under the assumptions of Theorem 1 (note that in the last four cases we also have that $\left.b_{3}= \pm\left(a_{2}-a_{3}\right)\right)$. This ends the proof of the lemma.

From Theorem 5 and Lemma 6 we have proved that in the case (i) the differential systems (2) either satisfy the conditions of Theorem 1, or have at most four functionally independent polynomial first integrals. Next we consider the case (ii).

In case (ii) $\Delta_{1} / A_{1}^{2}$ has the form $m_{1}^{2} n_{1}^{2} /\left(n_{1}+m_{1}\right)^{2}$ with $m, n \in \mathbb{Z} \backslash\{0\}$ coprime. So it follows from the expressions of $\Delta_{1}$ and $A_{1}$ that $\Delta_{1}$ should be a square of 
$\left(a_{2}-a_{1}\right) b_{2}\left(x_{2}^{2}+y_{2}^{2}\right)+\left(\left(a_{1}-a_{2}\right)^{2}+b_{2}^{2}-b_{1}^{2}\right) x_{2} y_{2}$ or of $\left(a_{2}-a_{3}\right) b_{2}\left(x_{2}^{2}+y_{2}^{2}\right)+\left(\left(a_{2}-\right.\right.$ $\left.\left.a_{3}\right)^{2}+b_{2}^{2}-b_{3}^{2}\right) x_{2} y_{2}$. Without loss of generality we can write it as

$$
\begin{aligned}
& \left(a_{2}-a_{1}\right) b_{2}\left(x_{2}^{2}+y_{2}^{2}\right)+\left(\left(a_{1}-a_{2}\right)^{2}+b_{2}^{2}-b_{1}^{2}\right) x_{2} y_{2} \\
& =L_{1}^{2}\left(\left(a_{2}-a_{3}\right) b_{2}\left(x_{2}^{2}+y_{2}^{2}\right)+\left(\left(a_{2}-a_{3}\right)^{2}+b_{2}^{2}-b_{3}^{2}\right) x_{2} y_{2}\right),
\end{aligned}
$$

and it is easy to check that $A_{1} /\left(\left(a_{2}-a_{3}\right) b_{2}\left(x_{2}^{2}+y_{2}^{2}\right)+\left(\left(a_{2}-a_{3}\right)^{2}+b_{2}^{2}-b_{3}^{2}\right) x_{2} y_{2}\right)$ is a constant. Set

$$
A_{1}=K_{1}\left(\left(a_{2}-a_{3}\right) b_{2}\left(x_{2}^{2}+y_{2}^{2}\right)+\left(\left(a_{2}-a_{3}\right)^{2}+b_{2}^{2}-b_{3}^{2}\right) x_{2} y_{2}\right) .
$$

Then, from (12) and (13) equating to zero the coefficients of the monomials in the variables $x_{2}$ and $y_{2}$ we have

$$
\begin{aligned}
-b_{2}\left(a_{1}-a_{2}+L_{1}^{2}\left(a_{2}-a_{3}\right)\right) & =0, \\
\left(a_{1}-a_{2}\right)^{2}-b_{1}^{2}+b_{2}^{2}-L_{1}^{2}\left(\left(a_{2}-a_{3}\right)^{2}+b_{2}^{2}-b_{3}^{2}\right) & =0, \\
\left(a_{1}-a_{2}\right)\left(a_{3}-a_{2}\right)+b_{2}^{2}+b_{2} K_{1}\left(a_{2}-a_{3}\right) & =0, \\
-2 b_{2}\left(a_{1}+a_{3}-2 a_{2}\right)+2 b_{1} b_{3}+K_{1}\left(\left(a_{2}-a_{3}\right)^{2}+b_{2}^{2}-b_{3}^{2}\right) & =0,
\end{aligned}
$$

where $L_{1} / K_{1}=m_{1} n_{1} /\left(n_{1}^{2}+m_{1}^{2}\right) \neq 0$.

Subcase (ii.1): If $K_{1}^{2}=4 L_{1}^{2}$, solving (14), using that $\left(b_{1}, b_{2}, b_{3}\right) \neq(0,0,0)$ and $b_{i}, a_{i} \in$ $\mathbb{R}$ for $i=1,2,3$ we obtain

$$
\begin{aligned}
& b_{1}= \pm\left(a_{2}-a_{1}\right), \quad b_{3}=0, \quad b_{2}=0, \quad a_{2}=a_{3} ; \\
& b_{1}=0, \quad b_{2}=0, \quad a_{1}=a_{2}, \quad b_{3}= \pm\left(a_{3}-a_{2}\right), \\
& b_{1}=b_{3} L_{1}, \quad a_{1}=a_{2}+b_{2} L_{1}, \quad a_{3}=a_{2}+\frac{b_{2}}{L_{1}} .
\end{aligned}
$$

Note that the first four conditions are inside the conditions of Theorem 1. Now we consider the last condition.

Lemma 7. The differential systems (2) under condition

$$
s_{5}=\left\{b_{1}=b_{3} L_{1}, a_{1}=a_{2}+b_{2} L_{1}, a_{3}=a_{2}+\frac{b_{2}}{L_{1}}\right\}
$$

either satisfy the conditions of Theorem 1, or the eigenvalues of the singularity $S_{2} d o$ not have a fifth linearly independent resonant lattice.

Proof. At the singularities $S_{2}$, the 6-tuple of eigenvalues of the linear part of the differential systems (2) are given in (7). Direct calculations show that under the condition $s_{5}$, the equation $\Delta_{2}=0$ yields that either $b_{2}=b_{3}=0$, which is not possible since otherwise $b_{i}=0$ for $i=1,2,3$, or $L_{1}^{2}=1$ and then $b_{1}=b_{3}, b_{2}=a_{1}-a_{2}=a_{3}-a_{2}$. Hence $a_{1}=a_{3}$. Thus, we are under the assumptions of Theorem 1 . Then, under condition $s_{5}$ either $\Delta_{2}=0$ and we are under the assumptions of Theorem 1 , or $\Delta_{2} \neq 0$. Now, working in a similar way as we did for the singularities $S_{1}$ for studying if there is a fifth linearly independent resonant lattice at $S_{1}$, we need to check if 
$\sqrt{A_{2}-\sqrt{B_{2}}} / \sqrt{A_{2}+\sqrt{B_{2}}} \neq 0$ is a rational number. For $S_{2}$ under the condition $s_{5}$ we have that

$$
\frac{\Delta_{2}}{A_{2}^{2}}=\frac{\left(L_{1}^{2}-1\right)\left(b_{3}^{2} L_{1}^{2} x_{1} y_{1}+b_{2}^{2}\left(L_{1}^{2}-1\right) x_{1} y_{1}+b_{2} b_{3} L_{1}^{2}\left(x_{1}^{2}+y_{1}^{2}\right)\right)^{2}}{L_{1}^{2}\left(4 b_{2} b_{3} L_{1}^{2} x_{1} y_{1}+b_{3}^{2} L_{1}^{2}\left(x_{1}^{2}+y_{1}^{2}\right)+b_{2}^{2}\left(L_{1}^{2}-1\right)\left(x_{1}^{2}+y_{1}^{2}\right)\right)^{2}} .
$$

Since $L_{1} \neq 0$ this shows that there always exist infinitely many singularities $S_{2}$ which cannot satisfy condition (8). At these singularities $S_{2}$ the eigenvalues do not have a fifth linearly independent resonant lattice.

From Theorem 5 and Lemma 7 we have proved that in the case (ii.1), the differential systems (2) either satisfy the conditions in Theorem 1 or have at most four functionally independent polynomial first integrals.

Subcase (ii.2): If $K_{1}^{2} \neq 4 L_{1}^{2}$, solving (14), using that $\left(b_{1}, b_{2}, b_{3}\right) \neq(0,0,0)$ and $b_{i}, a_{i} \in$ $\mathbb{R}$ for $i=1,2,3$ we obtain (note that $K_{1}^{2}-4 L_{1}^{2}=K_{1}^{2}\left(m_{1}-n_{1}\right)^{2}\left(m_{1}+n_{1}\right)^{2} /\left(m_{1}^{2}+n_{1}^{2}\right)^{2}>$ $0)$,

$$
\begin{aligned}
& b_{1}=\frac{b_{2}}{2}\left(2 \sigma_{1}+K_{1} \sigma_{2}+\sigma_{3} \sqrt{K_{1}^{2}-4 L_{1}^{2}}\right), \quad a_{1}=a_{2}+\frac{b_{2}}{2}\left(K_{1}+\sigma_{4} \sqrt{K_{1}^{2}-4 L_{1}^{2}}\right), \\
& b_{3}=\frac{b_{2}}{2 L_{1}^{2}}\left(\sigma_{5} K_{1}+2 \sigma_{6} L_{1}^{2}+\sigma_{7} \sqrt{K_{1}^{2}-4 L_{1}^{2}}\right), \quad a_{3}=a_{2}+\frac{b_{2}}{2 L_{1}^{2}}\left(K_{1}+\sigma_{8} \sqrt{K_{1}^{2}-4 L_{1}^{2}}\right) ; \\
& b_{1}=\mp \frac{2 L_{1}^{2}}{\sqrt{K_{1}^{2}-4 L_{1}^{2}}}\left(a_{2}-a_{3}\right), \quad a_{1}=a_{2}, \quad b_{3}=\mp \frac{K_{1}}{\sqrt{K_{1}^{2}-4 L_{1}^{2}}}\left(a_{2}-a_{3}\right), \quad b_{2}=0 ; \\
& b_{1}=\frac{b_{3} K_{1}}{2}, \quad a_{1}=a_{2} \mp \frac{b_{3}}{2} \sqrt{K_{1}^{2}-4 L_{1}^{2}}, \quad a_{3}=a_{2}, \quad b_{2}=0 ;
\end{aligned}
$$

with $\left(\sigma_{1}, \sigma_{2}, \sigma_{3}, \sigma_{4}, \sigma_{5}, \sigma_{6}, \sigma_{7}, \sigma_{8}\right)$ equal to

$$
\begin{aligned}
& (1,-1,1,-1,1,-1,-1,-1), \quad(1,1,-1,-1,1,1,-1,-1), \\
& (1,1,1,1,1,1,1,1), \quad(-1,-1,-1,1,-1,-1,-1,1) \\
& (-1,1,1,1,-1,1,-1,1), \quad(-1,-1,1,-1,-1,-1,1,-1), \\
& (1,-1,-1,1,1,-1,1,1), \quad(-1,1,-1,-1,-1,1,1,-1) .
\end{aligned}
$$

We remark that the second case is in fact four cases. We note that the first eight cases can be written, in particular, as one of the following four conditions

$$
\begin{array}{llll}
b_{1}=a_{2}-a_{1}+b_{2} & \text { and } & b_{3}=a_{3}-a_{2}-b_{2}, \\
b_{1}=a_{1}-a_{2}+b_{2} & \text { and } & b_{3}=a_{3}-a_{2}+b_{2}, \\
b_{1}=a_{2}-a_{1}-b_{2} & \text { and } & b_{3}=a_{2}-a_{3}-b_{2}, \\
b_{1}=a_{1}-a_{2}-b_{2} & \text { and } & b_{3}=a_{2}-a_{3}+b_{2} .
\end{array}
$$


These cases are all inside the conditions of Theorem 1 . Now we consider the six last conditions and we set

$$
\begin{gathered}
s_{6,7}=\left\{b_{1}=\mp \frac{2 L_{1}^{2}\left(a_{2}-a_{3}\right)}{\sqrt{K_{1}^{2}-4 L_{1}^{2}}}, \quad a_{1}=a_{2}, \quad b_{3}=-\frac{K_{1}\left(a_{2}-a_{3}\right)}{\sqrt{K_{1}^{2}-4 L_{1}^{2}}}, \quad b_{2}=0\right\}, \\
s_{8,9}=\left\{b_{1}=\mp \frac{2 L_{1}^{2}\left(a_{2}-a_{3}\right)}{\sqrt{K_{1}^{2}-4 L_{1}^{2}}}, \quad a_{1}=a_{2}, \quad b_{3}=\frac{K_{1}\left(a_{2}-a_{3}\right)}{\sqrt{K_{1}^{2}-4 L_{1}^{2}}}, \quad b_{2}=0\right\}, \\
s_{10,11}=\left\{b_{1}=\frac{b_{3} K_{1}}{2}, \quad a_{1}=a_{2} \mp \frac{b_{3}}{2} \sqrt{K_{1}^{2}-4 L_{1}^{2}}, \quad a_{3}=a_{2}, \quad b_{2}=0\right\} .
\end{gathered}
$$

Lemma 8. The differential systems (2) under one of the conditions $s_{6}, \ldots, s_{9}$ or $s_{10}$, $s_{11}$, either satisfy the conditions of Theorem 1, or the eigenvalues of the singularity $S_{2}$ do not have a fifth linearly independent resonant lattice.

Proof. At the singularities $S_{2}$, the 6-tuple of eigenvalues of the linear part of the differential systems (2) are given in (7). Now direct calculations show that under one of the conditions $s_{6}, \ldots, s_{9}$, the equation $\Delta_{2}=0$ yields $a_{2}=a_{3}$, which is not possible since otherwise $b_{i}=0$ for $i=1,2,3$. Then, under one of the conditions $s_{6}, \ldots, s_{9}$, we have $\Delta_{2} \neq 0$. Now working in a similar way as we did for the singularities $S_{1}$ for studying if there is a fifth linearly independent resonant lattice at $S_{2}$, we need to check if $\sqrt{A_{2}-\sqrt{B_{2}}} / \sqrt{A_{2}+\sqrt{B_{2}}} \neq 0$ is a rational number. Since on either $s_{6}, \ldots, s_{9}$ we have

$$
\left|\frac{\Delta_{2}}{A_{2}^{2}}\right|=\frac{L_{1}^{2}\left(K_{1}^{2}-4 L_{1}^{2}\right)\left(\sqrt{K_{1}^{2}-4 L_{1}^{2}}\left(x_{1}^{2}+y_{1}^{2}\right)+2\left(L_{1}^{2}-1\right) x_{1} y_{1}\right)}{2\left(-K_{1}^{2} x_{1} y_{1}+L_{1}^{2}\left(\sqrt{K_{1}^{2}-4 L_{1}^{2}}\left(x_{1}^{2}+y_{1}^{2}\right)+4 x_{1} y_{1}\right)\right)^{2}} x_{1} y_{1},
$$

and since $L_{1}^{2}\left(K_{1}^{2}-4 L_{1}^{2}\right) \neq 0$ this shows that there always exist infinitely many singularities $S_{2}$ which cannot satisfy condition (8). At these singularities $S_{2}$ the eigenvalues do not have a fifth linearly independent resonant lattice.

Now direct calculations show that under one of the conditions $s_{10}, s_{11}$ using that $K_{1}^{2} \neq 4 L_{1}^{2}$, the equation $\Delta_{2}=0$ yields $b_{3}=0$ which is not possible since otherwise $b_{i}=0$ for $i=1,2,3$. Then, under one the conditions $s_{10}, s_{11}$ we have $\Delta_{2} \neq 0$. Now working in a similar way as we did for the cases $s_{6}, \ldots, s_{9}$, we must have that under one of the conditions $s_{10}, s_{11}$, condition (8) must hold. However, since $\Delta_{2} / A_{2}^{2}=N_{1} N_{2} / D_{1}$ with

$$
\begin{aligned}
& N_{1}=K_{1} \sqrt{K_{1}^{2}-4 L_{1}^{2}}\left(x_{1}^{2}+y_{1}^{2}\right)+2\left(K_{1}^{2}-2 L_{1}^{2}\right) x_{1} y_{1}, \\
& N_{2}=K_{1} \sqrt{K_{1}^{2}-4 L_{1}^{2}}\left(x_{1}^{2}+y_{1}^{2}\right)+2\left(K_{1}^{2}-2 L_{1}^{2}-2\right) x_{1} y_{1}, \\
& D_{1}=4\left(\left(K_{1}^{2}-2 L_{1}^{2}\right)\left(x_{1}^{2}+y_{1}^{2}\right)+2 K_{1} \sqrt{K_{1}^{2}-4 L_{1}^{2}} x_{1} y_{1}\right)^{2},
\end{aligned}
$$

and since $L_{1} K_{1}\left(K_{1}^{2}-4 L_{1}^{2}\right) \neq 0$ this shows again that there always exist infinitely many singularities $S_{2}$ which cannot satisfy condition (8). At these singularities $S_{2}$ the eigenvalues do not have a fifth linearly independent resonant lattice. 
From Theorem 5 and Lemma 8 we have proved that in the case (ii.2), the differential systems (2) either satisfy the conditions in Theorem 1 or have at most four functionally independent polynomial first integrals.

In short, if cases (i) or (ii) hold then the conditions given in Theorem 1 are satisfied and by Corollary 2 the differential systems (2) are completely integrable. If cases (i) and (ii) do not hold then by Theorem 5 the differential systems (2) can have at most four analytic integrals in a neighborhood of a point of $S$. Consequently the differential systems (2) have at most four functionally independent polynomial first integrals. This completes the proof of Theorem 3

\section{ACKNOWLEDGEMENTS}

The first author was partially supported by the MICINN/FEDER grant MTM200803437, AGAUR grant 2009SGR-410 and ICREA Academia. The second author was supported by AGAUR grant PIV-DGR-2010 and by FCT through the project PTDC/MAT/117106/2010 and through CAMGSD, Lisbon.

\section{REFERENCES}

[1] W. Cong, J. Llibre And X. Zhang, Generalized rational first integrals of analytic differential systems, J. Differential Equations 251 (2011), 2770-2788.

[2] A. GoRiely, Integrability and nonintegrability of dynamical systems, Advanced Series in Nonlinear Dynamics 19, World Sci. Publ. Co., Inc. River Edge, NJ, 2001.

[3] Y.N. Fedorov and V.V. Kozlov, Various Aspects of $n$-Dimensional Rigid Body Dynamics. In Dynamical Systems in Classical Mechanics, Ed. V.V. Kozlov, Am. Math. Soc. Translations, series 2, 1991. V. 168.

[4] J. Llibre, S. Walcher and X. Zhang, Local Darboux first integrals of analytic differential systems, preprint.

[5] S.V. Manakov, A remark on the integration of the Eulerian equations of the dynamics of an n-dimensional rigid body, Funkcional Anal. i Prilozen. V. 6 (1972), 83-84. English transl. Funct. Anal. Appl. 10 (1977), 328-329.

[6] P. NegRinI, Integrability, nonintegrability and chaotic motions for a system motivated by the Riemann ellipsoids problem, Regul. Chaotic Dyn. 8 (2003), 349-374.

[7] X. Zhang, Local first integrals for systems of differential equations, J. Phys. A 36 (2003), $12243-12253$.

${ }^{1}$ Departament de Matemàtiques, Universitat Autònoma de Barcelona, 08193 Bellaterra, Barcelona, Catalonia, Spain

E-mail address: jllibre@mat.uab.cat

2 Departamento de Matemática, Instituto Superior Técnico, Universidade Técnica de Lisboa, Av. Rovisco Pais 1049-001, Lisboa, Portugal

E-mail address: cvalls@math.ist.utl.pt 